\title{
Tratamiento de la neumonía del adulto adquirida en la comunidad
}

\author{
ALEJANDRO DÍAZ F., JAIME LABARCA L., CARLOS PÉREZ C., \\ MAURICIO RUIZ C. y MARCELO WOLFF R.
}

\section{Treatment of community-acquired pneumonia in adults}

Appropriate antibiotic treatment reduces the duration of symptoms associated to pneumonia, the risk of complications and mortality. In most cases, it is not possible to identify the etiologic agent so antibiotic treatment is empirically prescribed. In Chile, one third of Streptococcus pneumoniae strain isolates has diminished susceptibility to penicillin; in-vitro erythromycin resistance is about $10-15 \%$ and cefotaxime resistance $2-10 \%$. It is recommended to classify patients with community acquired pneumonia in four risk categories: Group 1: patients under 65 years without co-morbidities, in ambulatory attendance. Treatment: oral amoxicillin $1 \mathrm{~g}$ TID, 7 days. Group 2: patients over 65 years and / or co-morbidities, in ambulatory attendance. Treatment: oral amoxicillin/clavulanate 500/125 mg TID or $875 / 125 \mathrm{mg}$ BID, or cefuroxime $500 \mathrm{mg}$ BID, 7 days. Group 3: patients admitted to general wards with criteria of moderate severity. Treatment: ceftriaxone 1-2 g once a day or cefotaxime $1 \mathrm{~g}$ TID, IV, 7-10 days. Group 4: patients with severe CAP that must be interned into ICU. Treatment: ceftriaxone $2 \mathrm{~g}$ once a day or cefotaxime $1 \mathrm{~g}$ TID, IV, associated to erythromycin 500 QID, levofloxacin 500-1.000 mg once a day, or moxifloxacin $400 \mathrm{mg} /$ once a day, IV, 10-14 days. In the presence of allergy to or treatment failure with betalactam drugs and/or positive serology for Mycoplasma, Chlamydia or Legionella sp it is recommended to add: erythromycin 500 mg QID, IV or oral, oral clarithromycin $500 \mathrm{mg}$ BID, or oral azythromycin $500 \mathrm{mg}$ once a day.

Key words: pneumonia, treatment, disease management, drug therapy.

Palabras claves: neumonía, tratamiento, manejo de la enfermedad, antibióticos.

\section{Introducción}

El tratamiento antibiótico apropiado reduce la duración de la sintomatología asociada a la neumonía, el riesgo de complicaciones y la mortali$\operatorname{dad}^{1}$. En la mayoría de los casos, no es posible identificar el agente microbiológico que ocasiona la infección y por esto el tratamiento antibiótico se prescribe en forma empírica. Esto constituye la práctica habitual en el manejo de los pacientes ambulatorios y hospitalizados por neumonía adquirida en la comunidad (NAC), el tratamiento empírico rara vez se modifica sobre la base de los resultados microbiológicos. En los últimos años varias sociedades científicas han elaborado guías clínicas para el manejo de la NAC del adulto ${ }^{1-4}$. Algunos estudios sugieren que la implementación de las guías clínicas para el tratamiento de la NAC acorta el tiempo de hospitalización ${ }^{5}$, el uso de tratamiento antibiótico endovenoso ${ }^{5}$ y disminuye el riesgo de complicaciones y muerte 6,7 . En una comunicación reciente, cerca de la mitad de los pacientes internados en un hospital universitario por NAC fueron manejados siguiendo las recomendaciones de la guía clínica de la Sociedad Chilena de Enfermedades Respiratorias ${ }^{8}$. De este modo, es necesario destacar la importancia de la difusión de las guías clínicas actualizadas en el medio ambulatorio y hospitalario de nuestro país, con-

Facultad de Medicina. Pontificia Universidad Católica de Chile, Santiago, Chile.

Departamento de Enfermedades Respiratorias, (ADF).

Departamento Medicina, (JLL, CPC).

Sección Enfermedades Respiratorias, Hospital Clínico Universidad de Chile, Santiago, Chile (MRC).

Servicio de Medicina, Hospital Clínico San Borja-Arriarán, Universidad de Chile, Santiago, Chile (MWR). 
siderando la información epidemiológica y los estudios clínicos realizados en el medio nacional.

Los antecedentes epidemiológicos, la presencia de comorbilidad y la estimación de la gravedad son los principales factores que determinan el pronóstico del paciente con NAC y por lo tanto deben ser considerados para decidir el lugar de manejo y la elección del tratamiento antimicrobiano empírico ${ }^{2,9}$. Otra variable a considerar, es el conjunto de patógenos posibles que pueden ocasionar el episodio de neumonía, y esto es especialmente relevante en determinados contextos epidemiológicos para orientar el tratamiento (ej: brote de influenza en el periodo otoño-invierno, infección por hantavirus en el sur de Chile o por Legionella sp en determinados ambientes).

\section{Resistencia de Streptococcus pneumoniae a los antibióticos}

La resistencia a antibióticos de Streptococcus pneumoniae, el patógeno más frecuente que causa neumonía, ha ido en aumento en todo el mundo. Según el estudio Sentry realizado en el periodo 1997-2001, en Chile un tercio de las cepas de $S$. pneumoniae muestra susceptibilidad disminuida a penicilina y en el $18 \%$ de los casos la resistencia es alta (concentración inhibitoria mínima $(\mathrm{CIM}) \geq 2 \mu \mathrm{g} / \mathrm{ml})$; mientras que la resistencia a eritromicina es cercana al $12 \%$ y a cefotaxima $2 \%^{10}$. En un estudio reciente, el patrón de resistencia de $S$. pneumoniae aislado de procesos invasores y no en un hospital universitario de Santiago mostró susceptibilidad disminuida a penicilina en $31 \%$ de los casos, a macrólidos en $21 \%$ y a cefotaxima en $16 \%{ }^{11}$. La evolución temporal de la resistencia de $S$. pneumoniae a antimicrobianos se describe en la Figura 1. En 1999 no se encontraron cepas de $S$. pneumoniae resistente a levofloxacina ${ }^{12}$; sin embargo, en un informe del año 2003 la resistencia alcanzó a $9 \%{ }^{13}$. En adultos con neumonía neumocócica, las cepas con susceptibilidad disminuida a penicilina han fluctuado entre $5 \%^{14}$ y $16 \%{ }^{15}$; la resistencia a eritromicina entre $1,3 \% \%^{15}$ y $15 \%{ }^{14}$; y a cefalosporinas de tercera generación entre $0 \%{ }^{14}$ y $8 \%{ }^{15}$. El patrón de resistencia a antimicrobianos de $S$. pneumoniae varía según el área geográfica, población (infantil o adulta) y tipo de procesos (invasores y no invasores) examinados.

La implicancia clínica de la resistencia a antibióticos de $S$. pneumoniae es controvertida, ya que algunos estudios demostraron un incre- mento significativo de la mortalidad ${ }^{16,17}$, pero cuando estudios posteriores ajustaron la letalidad a otros factores de riesgo, la resistencia a penicilina con niveles de CIM entre 0,1 y $2 \mu \mathrm{g} / \mathrm{ml}$ no implicaron un mayor riesgo de complicaciones y muerte ${ }^{17,18}$. El estudio de Feikin y cols encontró un aumento de la letalidad en pacientes con neumonía neumocócica con CIM $\geq 4 \mu \mathrm{g} / \mathrm{ml}^{18}$. Afortunadamente, no se han comunicado cepas con este nivel de resistencia en la población adulta de nuestro país ${ }^{10-15}$.

Varios estudios extranjeros han demostrado la eficacia de los agentes $\beta$-lactámicos ${ }^{16,17,19}$ en el tratamiento de la neumonía neumocócica con CIM para penicilina inferior a $4 \mu \mathrm{g} / \mathrm{ml}$ [Ib]. Los escasos estudios efectuados en el medio nacional en muestras pequeñas de pacientes corroboran los hallazgos de las grandes series ${ }^{15,20}$ [II]

El conjunto de la información sugiere que la resistencia a antimicrobianos en pacientes adultos con neumonía neumocócica aún no es un hecho muy frecuente en nuestro medio, los agentes $\beta$-lactámicos siguen siendo efectivos y no hay certeza que exista mayor riesgo de muerte o falla clínica asociado a la resistencia ${ }^{10-17}$. Sin embargo, la emergencia de cepas de $S$. pneumoniae resistentes a antimicrobianos en nuestro medio es una realidad, se ha asociado al uso indiscriminado de antibióticos en la patología respiratoria de origen viral, especialmente en la población pediátrica, y consideramos que es responsabilidad de los médicos educar a la población sobre este tema y prescribir los antimicrobianos racionalmente, para evitar el incremento sostenido de este problema en nuestro medio [D].

\section{Impacto de la elección de los antibióticos en la resistencia}

El uso amplio de agentes $\beta$-lactámicos y nuevas fluoroquinolonas se ha asociado a un aumento de la resistencia a antimicrobianos de los principales patógenos respiratorios, especialmente en el medio hospitalario. El incremento de la prescripción de cefalosporinas de tercera generación se ha asociado a un aumento de la diarrea por Clostridium difficile ${ }^{21}$, la aparición de cepas de Klebsiella pneumoniae productoras de $\beta$-lactamasas de espectro extendido ${ }^{22}$, y el desarrollo de cepas resistentes a cefotaxima (62\%) y ceftazidima $(45 \%)^{23}$. El uso de fluoroquinolonas y aminoglucósidos constituye factor de riesgo independiente para el desarrollo de cepas de Escherichia coli y $K$. pneumoniae resistentes en el medio hospitalario. La exposición a fluoroqui- 


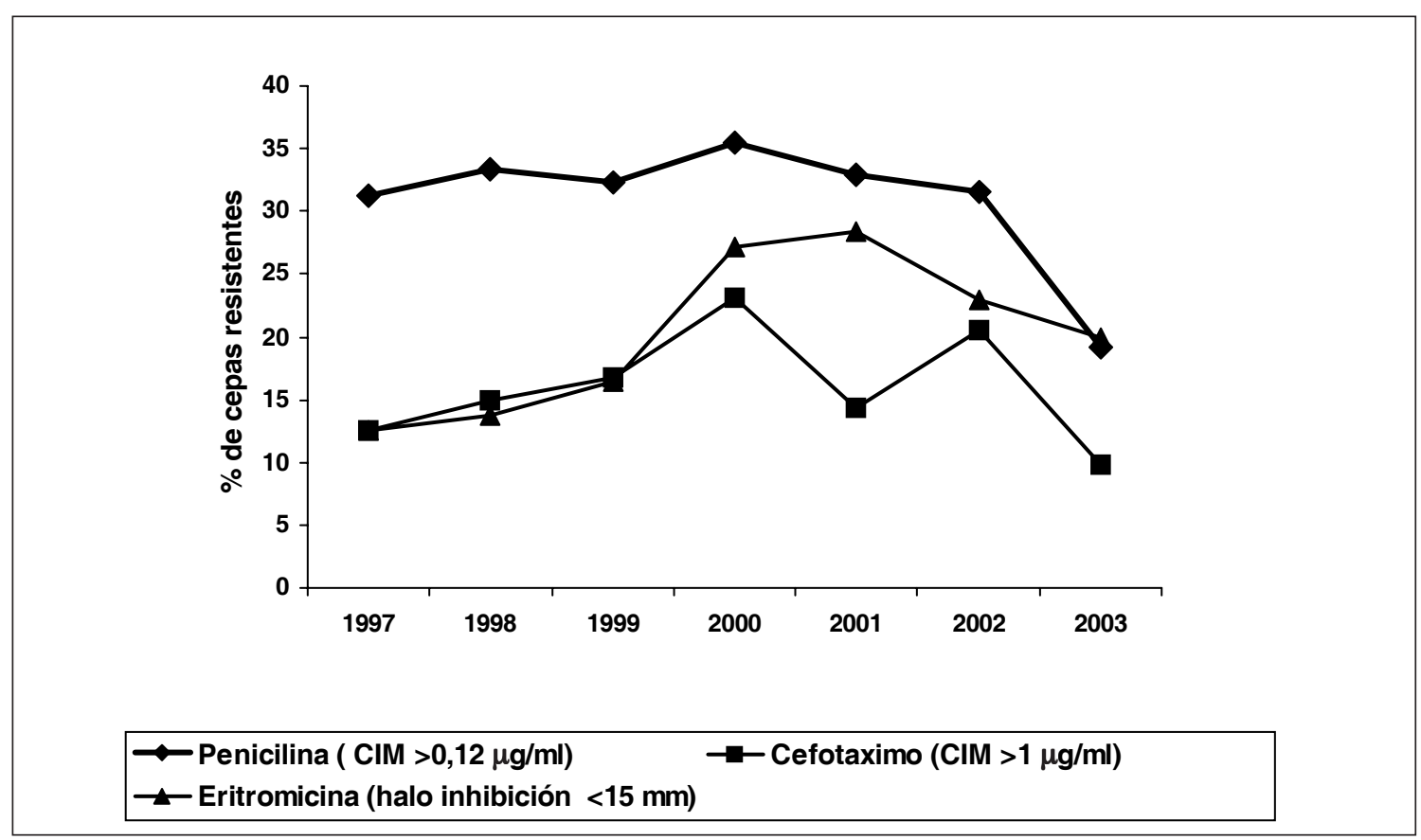

Figura 1. Resistencia a penicilina, eritromicina y cefotaxima de 901 cepas de S. pneumoniae, Santiago de Chile, $1997-$ 2003 (11).

nolonas se ha asociado a un riesgo 3 a 4 veces mayor de desarrollar bacteriemias nosocomiales por $S$. aureus meticilina-resistente (SAMR) ${ }^{24}$.

El impacto negativo que conlleva el uso inapropiado de antimicrobianos, especialmente en el ambiente ambulatorio, es básicamente responsabilidad de los médicos y se recomienda tomar medidas con el propósito de racionalizar la prescripción de antibióticos, y así reducir la selección de cepas resistentes [C].

\section{Nuevos antibióticos para las infecciones respiratorias}

La nuevas quinolonas o fluoroquinolonas están siendo utilizadas en el manejo de diversas infecciones respiratorias ${ }^{25}$ y no fueron mencionadas en la primera guía clínica de la Sociedad Chilena de Enfermedades Respiratorias9. Las fluoroquinolonas son muy activas contra $S$. pneumoniae sensible o resistente a $\beta$-lactámicos y macrólidos; además, son activas frente a $\mathrm{Myco-}$ plasma pneumoniae, Chlamydia pneumoniae y Legionella pneumophila, y contra los bacilos gramnegativos entéricos. Estos antibióticos tienen alta biodisponibilidad oral y la mayoría pueden administrarse una vez al día por vía oral o endovenosa ${ }^{25}$. En Chile, se encuentran disponibles para la administración oral: levofloxacina, moxifloxacina y gatifloxacina. Las dos primeras también se encuentran en forma inyectable y ambas se indican una vez al día y pueden inyectarse por una vía periférica. En nuestro medio se ha observado un rápido incremento de la resistencia a levofloxacina asociado a su prescripción indiscriminada ${ }^{13}$, y existe escasa información acerca de las otras fluoroquinolonas. Varias guías clínicas han incluido a estos agentes en sus recomendaciones y la razón fundamental ha sido la alta tasa de resistencia de $S$. pneumoniae a penicilina y macrólidos observada en algunas áreas geográficas y el espectro de cobertura antimicrobiano que incluye a los agentes atípicos $^{1-4}$. Sin embargo, este grupo de antibióticos altamente efectivos debiera reservarse para pacientes hospitalizados con NAC grave y sólo se recomendaría su uso en casos muy seleccionados [C]. Esta recomendación se basa en los siguientes antecedentes: la resistencia de $S$. pneumoniae a agentes $\beta$-lactámicos con CIM inferior a $4 \mu \mathrm{g} / \mathrm{ml}$ no se ha asociado a mayor riesgo de complicaciones y muerte [II]; en Chile no se han detectado cepas resistentes a penicilina con CIM $>4 \mu \mathrm{g} / \mathrm{ml}$ en adultos con neumonía comunitaria [II]; en nuestro medio, el manejo con agentes $\beta$-lactámicos y macrólidos de la NAC neumocócica ocasionada por cepas resistentes en adultos sigue siendo una conducta segura y eficaz [B], y en el medio ambulatorio se considera apropiado racionalizar la prescrip- 
ción de antibióticos de amplio espectro con el propósito de reducir la selección de cepas resistentes [D].

\section{Fundamentos de las recomendaciones de tratamiento}

Las recomendaciones de esta guía se han basado en información actualizada acerca de la resistencia a antibióticos de los principales patógenos respiratorios, especialmente de $S$. pneumoniae, tanto en el medio nacional como extranjero; el posible impacto de los antimicrobianos, especialmente los agentes de amplio espectro, sobre la selección de cepas resistentes; la información disponible sobre agentes antimicrobianos individuales, estudios de costoefectividad y la disponibilidad de medicamentos en el medio nacional.

Las recomendaciones de tratamiento se han formulado clasificando a los pacientes con NAC en cuatro categorías de riesgo de acuerdo a las siguientes variables clínicas: la edad del enfermo (se considera adulto mayor a aquel de 65 años o más); la presencia de comorbilidades específicas; la estimación de la gravedad en el momento de la evaluación inicial, y el lugar de manejo (ambulatorio, sala de cuidados generales o unidad de cuidados intensivos (UCI)) [C]. Para cada categoría se describen los principales agentes etiológicos de la neumonía ${ }^{9}$ (ver etiología, página 79) y las recomendaciones de tratamiento antimicrobiano de primera elección y esquemas alternativos.

Los pacientes con NAC se han agrupado en cuatro categorías de riesgo:

Grupo 1: pacientes menores de 65 años sin comorbilidad de manejo ambulatorio.

Grupo 2: pacientes mayores de 65 años y/o con comorbilidad de manejo ambulatorio.

Grupo 3: pacientes hospitalizados en sala de cuidados generales que tienen criterios de gravedad moderada.

Grupo 4: pacientes con NAC grave que deben ser manejados en la Unidad de Cuidados Intermedios o UCI.

Si un paciente presenta criterios de neumonía grave (ver NAC grave, página 108), aunque no pueda hospitalizarse en una unidad de cuidado crítico, debe manejarse como un paciente de alto riesgo debido a que la letalidad en esta categoría es superior al $20 \%{ }^{26}$ [C].

\section{Tratamiento antimicrobiano de la NAC de atención ambulatoria}

Los pacientes seleccionados apropiadamente para manejo ambulatorio tienen bajo riesgo de complicaciones y la letalidad es inferior al 1$2 \%{ }^{27}$, pero en Chile no existe información epidemiológica específica acerca de este grupo. En esta categoría se reconoce a dos grupos de pacientes: Grupo 1: adultos menores de 65 años sin comorbilidad y, Grupo 2: adultos mayores o de cualquier edad con comorbilidad específica. Los agentes etiológicos más frecuentes son $S$. pneumoniae, M. pneumoniae, C. pneumoniae y los virus respiratorios ${ }^{28-30}$ [Ib]. Una amplia variedad de antibióticos son eficaces en pacientes con NAC manejados en el medio ambulatorio y esta respuesta parece ser independiente del agente etiológico. La eficacia clínica de amoxicilina oral en dosis elevadas (mayor a $2 \mathrm{~g} /$ día) ) $^{31-35}$ [I $\left.\mathbf{I b}\right]$, los agentes $\beta$-lactámicos asociados a un inhibidor de $\beta$-lactamasa ${ }^{36}$ [Ib], los macrólidos y azálidos (eritromicina, claritromicina y azitromicina) ${ }^{37-41}$ [Ib] y las cefalosporinas de $2^{\text {a }}$ generación (cefuroxima $)^{42-44}$ [I Ib], ha sido demostrada en varios estudios clínicos que incluyen pacientes ambulatorios y hospitalizados. Además, un meta-análisis reciente ha confirmado que el tratamiento de la neumonía ambulatoria con eritromicina, claritromicina y una nueva fluoroquinolona tienen eficacia similar ${ }^{44}$ [Ia].

Basados en estos antecedentes, se recomienda que el tratamiento antibiótico empírico de la neumonía de atención ambulatoria deba cubrir fundamentalmente a $S$. pneumoniae, y ocasionalmente sea necesario cubrir los llamados agentes atípicos (Mycoplasma sp, Chlamydia sp y Legionella sp) [C]. Los antibióticos recomendados se describen en la Tabla 1. En pacientes del grupo 1 se recomienda el uso de amoxicilina en dosis elevada ( $3 \mathrm{~g} /$ día) y como alternativa, o en caso de alergia a $\beta$-lactámicos, prescribir un macrólido o azálido tales como eritromicina, claritromicina o azitromicina [C]. En el grupo 2, que tiene mayor riesgo de complicaciones, se recomienda el uso de amoxicilina/ácido clavulánico o cefuroxima. Como régimen alternativo, o en caso de alergia a los $\beta$-lactámicos, se recomienda prescribir un macrólido o azálido tales como eritromicina, claritromicina o azitromicina [D].

Recomendaciones para pacientes de bajo riesgo, de manejo ambulatorio

- Ante la sospecha clínica de una neumonía se sugiere confirmar el diagnóstico con una radiografía de tórax; si no es posible obtenerla, se sugiere tratar al paciente como si tuviera una infección pulmonar porque el pronóstico del paciente empeora cuando se retrasa el inicio del tratamiento antibiótico [C].

- Es importante aplicar una evaluación objetiva 
de la gravedad y determinar si existe algún criterio de hospitalización (ver evaluación de la gravedad, página 103). El manejo ambulatorio de un paciente con neumonía implica la ausencia de criterios clínicos y/o sociales de riesgo que recomienden su hospitalización [C].

- Cuando sea factible, la evaluación del paciente debe considerar la medición de la saturación arterial de oxígeno mediante oximetría de pulso, y si la $\mathrm{SaO}_{2}$ es inferior a $90 \%$, se recomienda derivar el enfermo al hospital para corregir la insuficiencia respiratoria [C].

- El antibiótico prescrito debe ser administrado precozmente, idealmente antes de 8 horas de realizado el diagnóstico [D].

- El paciente debe ser enviado a su domicilio con indicación de reposo, control de temperatura, hidratación oral e inicio del tratamiento antimicrobiano vía oral [D].

- El paciente debe acudir a control al finalizar el tratamiento antimicrobiano, o inmediatamente, en caso de evolución desfavorable: persistencia de la fiebre por más de tres días, aumento de la dificultad respiratoria o compromiso del estado general, aparición de criterios de gravedad (ver evaluación de la gravedad, página 101) [C].

- El tratamiento antimicrobiano prescrito debe durar siete días, excepto si se indica azitromicina, en cuyo caso bastarían cinco días [C].

\section{Tratamiento antimicrobiano de la NAC manejada en el hospital, en la sala de cuidados generales}

Los pacientes con NAC admitidos al hospital, manejados en la sala de cuidados generales, tienen un riesgo intermedio de complicaciones y muerte comparado con aquellos tratados en el medio ambulatorio y los internados en unidades de cuidado crítico $^{1-4}$. En Chile, la letalidad de los pacientes con NAC del grupo 3 varía entre 3 y $20 \%{ }^{45,46}$. Los patógenos respiratorios más relevantes en esta categoría son $S$. pneumoniae, Haemophilus influenzae, los microorganismos atípicos (M. pneumoniae, C. pneumoniae y Legionella $\mathrm{sp}$ ), los bacilos gramnegativos entéricos y los anaerobios estrictos ${ }^{1-4}$. En un estudio reciente realizado en Santiago, se demostró que $S$. pneumoniae era el agente más frecuente seguido de los virus influenza y parainfluenza ${ }^{15}$ [II]. Los agentes atípicos representaron cerca del $15 \%$ de los casos con etiología demostrada.

El tratamiento de la NAC que requiere hospi- talización con cefalosporinas de $2^{\mathrm{a}}$ y $3^{\mathrm{a}}$ generación (cefuroxima, cefotaxima, ceftriaxona), y los agentes $\beta$-lactámicos con inhibidor de $\beta$ lactamasa (amoxicilina/ácido clavulánico, ampicilina/sulbactam), han demostrado ser costoefectivos $^{36,42,43,47}$ [II]. Aún persiste la controversia sobre cuándo los pacientes con NAC de gravedad intermedia, hospitalizados en sala, deben recibir tratamiento con un agente $\beta$-lactámico, la combinación de un $\beta$-lactámico y un macrólido, o una fluoroquinolona. Algunos estudios retrospectivos han demostrado que la terapia combinada o con fluoroquinolonas reduciría la tasa de complicaciones, la estadía en el hospital y la letalidad comparado con el tratamiento con un agente $\beta$-lactámico [III]. En un meta-análisis reciente se examinaron ocho artículos que comparaban el tratamiento con un agente $\beta$ lactámico sólo o asociado a macrólidos o una nueva fluoroquinolona sola en adultos hospitalizados por $\mathrm{NAC}^{48}$. En seis estudios se encontró una reducción significativa de la letalidad en el hospital, en dos estudios disminuyó la estadía hospitalaria y en otro no hubo beneficio de la terapia combinada o fluoroquinolona sola sobre la monoterapia con $\beta$-lactámicos [III]. El diseño de todos los estudios fue descriptivo observacional no experimental, la mayoría fueron retrospectivos y los resultados podrían estar influenciados por factores de riesgo no controlados y por error en la selección de los pacientes para indicar un régimen u otro. Además, las variables clínicas medidas demostraron algunas inconsistencias entre los distintos estudios. En conclusión, aún está pendiente la ejecución de un ensayo clínico controlado que permita superar las fallas metodológicas n- 
Tabla 1. Recomendaciones de tratamiento antimicrobiano empírico para pacientes con neumonía adquirida en la comunidad manejados en el medio ambulatorio

\begin{tabular}{lll}
\hline Categoría de la neumonía & $\begin{array}{l}\text { Antibiótico de elección, dosis, } \\
\text { vía y duración del tratamiento }\end{array}$ & Régimen alternativo \\
\hline $\begin{array}{l}\text { Grupo 1: pacientes con } 65 \text { años } \\
\text { o menos, sin comorbilidad y } \\
\text { factores de riesgo }\end{array}$ & $\begin{array}{l}\text { Amoxicilina } 1 \mathrm{~g} \text { cada } 8 \text { horas } \\
\text { vía oral por } 7 \text { días }\end{array}$ & $\begin{array}{l}\text { Eritromicina } 500 \mathrm{mg} \text { cada } 6 \text { horas vía } \\
\text { oral, por } 7 \text { días, claritromicina } 500 \mathrm{mg} \\
\text { cada } 12 \text { horas, vía oral por } 7 \text { días, o } \\
\text { azitromicina } 500 \text { mg cada } 24 \text { horas, vía } \\
\text { oral por } 5 \text { días }\end{array}$ \\
\end{tabular}

Grupo 2: pacientes mayores de 65 años o de cualquier edad con comorbilidad específica
Amoxicilina/ácido clavulánico 500/ $125 \mathrm{mg}$ cada 8 horas u $875 / 125 \mathrm{mg}$ cada 12 horas vía oral por 7 días, o cefuroxima $500 \mathrm{mg}$ cada 12 horas vía oral por 7 días
Eritromicina 500 mg cada 6 horas, vía oral por 7 días, claritromicina $500 \mathrm{mg}$ cada 12 horas, vía oral, por 7 días, o azitromicina $500 \mathrm{mg}$ cada 24 horas, vía oral, por 5 días

\footnotetext{
* En caso de alergia a $\beta$-lactámicos se recomienda usar macrólidos.

** En pacientes con intolerancia digestiva a eritromicina, se sugiere utilizar claritromicina o azitromicina.
}

nerales (grupo 3). El esquema antibiótico debe ser administrado por vía endovenosa. En esta categoría, el tratamiento antimicrobiano de elección es una cefalosporina de $3^{\text {a }}$ generación sola (cefotaxima o ceftriaxona) [A-]. El régimen alternativo es amoxicilina/ácido clavulánico o ampicilina/sulbactam. Cuando en el hospital se ha demostrado un incremento en la tasa de patógenos nosocomiales resistentes a antimicrobianos asociado al empleo de cefalosporinas, se recomienda utilizar aminopenicilinas asociadas a un inhibidor de $\beta$-lactamasa [D]. La adición de eritromicina, claritromicina o azitromicina se debe reservar para los pacientes que no responden al tratamiento empírico inicial en el hospital, aquellos con riesgo epidemiológico conocido o que tienen una NAC grave [D].

Dos estudios descriptivos han demostrado una reducción de la letalidad cuando el tratamiento antibiótico es administrado antes de las 8 horas de la admisión al hospital ${ }^{53,54}$ [III]; también se ha comunicado una reducción de la estadía hospitalaria cuando el antibiótico se administra en el Servicio de Urgencia ${ }^{55}$ [III]. De este modo, se recomienda iniciar la terapia antibiótica precozmente, idealmente dentro de las 8 horas de ingresado el enfermo al hospital, y esto se logra fácilmente cuando el antibiótico se administra en el Servicio de Urgencia. Los hemocultivos deben tomarse antes de la administración de cualquier antibiótico y no deben retrasar el inicio del tratamiento [D].

En dos estudios etiológicos realizados en el medio nacional se ha comunicado la participación de los virus respiratorios en pacientes con $\mathrm{NAC}^{15,50}$ [II]. Sobre la base de esta información, se recomienda la búsqueda sistemática de agentes virales durante las épocas de alta circulación de estos microorganismos en la comunidad [D]. En la práctica clínica se recomienda que los pacientes infectados por virus influenza A o B sean aislados en cohorte para evitar la propagación nosocomial. En la infección por virus influenza A se recomienda el uso de amantadina $100 \mathrm{mg}$ cada 12 horas durante una semana $^{51}$ [C]. En los mayores de 65 años y portadores de insuficiencia renal crónica la dosis se debe reducir a $100 \mathrm{mg}$ por día. Además, los inhibidores de la neuraminidasa (zanamivir, oseltamivir) se recomiendan para el tratamiento de la influenza $\mathrm{A} \mathrm{y}^{52}$ [C]. Para los otros virus respiratorios aún no se dispone de un tratamiento antiviral eficaz.

Recomendaciones para pacientes con NAC de riesgo moderado hospitalizados en sala

- En el servicio de urgencia se debe evaluar la gravedad del enfermo y medir la saturación arterial de oxígeno con oximetría de pulso, si la $\mathrm{SaO}_{2}$ es inferior a $90 \%$ se debe corregir la insuficiencia respiratoria administrando oxígeno por naricera o mascarilla y se debe efectuar la medición de gases arteriales [C].

- Solicitar dos hemocultivos antes de iniciar el tratamiento antimicrobiano [C].

- Realizar detección rápida de virus respiratorios de hisopado o aspirado nasofaríngeo durante la época de alta circulación de virus en la comunidad [D].

- El antibiótico prescrito debe ser administrado precozmente, idealmente antes de 8 horas de realizado el diagnóstico [C].

- Si se detecta infección por virus influenza A o B se debe indicar aislamiento respiratorio en cohorte y prescribir terapia antiviral [C]. 
Tabla 2. Recomendaciones de tratamiento antimicrobiano empírico para pacientes con neumonía adquirida en la comunidad manejados en el hospital

\begin{tabular}{ll}
\hline Categoría de la neumonía & $\begin{array}{l}\text { Antibiótico de elección, dosis, } \\
\text { vía y duración del tratamiento }\end{array}$ \\
\hline $\begin{array}{l}\text { Grupo 3: pacientes de cualquier } \\
\text { edad, con criterios de gravedad } \\
\text { moderada, hospitalizados en } \\
\text { sala de cuidados generales }\end{array}$ & $\begin{array}{l}\text { Ceftriaxona 1-2 gramos/día EV, o } \\
\text { cefotaxima } 1 \text { gramo cada } 8 \text { horas EV }\end{array}$ \\
& \\
& En presencia de: \\
& - Fracaso de tratamiento con agentes \\
& $\beta$-lactámicos (definición en el texto). \\
& - Serología positiva para Mycoplasma sp, \\
Chlamydia sp o Legionella sp
\end{tabular}

Se recomienda agregar:

Eritromicina $500 \mathrm{mg}$ cada $6 \mathrm{~h} \mathrm{EV} \mathrm{o} \mathrm{VO,}$ claritromicina $500 \mathrm{mg}$ cada $12 \mathrm{~h} \mathrm{VO}$, o azitromicina $500 \mathrm{mg} /$ día VO

Grupo 4: pacientes con NAC grave hospitalizados en la Unidad de Intermedio o UCI

\section{Régimen alternativo}

Amoxicilina/ácido clavulánico 1.000/

$200 \mathrm{mg} \mathrm{c} / 8 \mathrm{~h} \mathrm{EV}$, amoxiclina/sulbactam

$1.000 / 500 \mathrm{mg}$ cada $8 \mathrm{~h} \mathrm{EV}$, o ampicilina/sulbactam 1.000/500 mg cada $8 \mathrm{~h} \mathrm{EV}$

En presencia de:

- Fracaso de tratamiento con agentes

$\beta$-lactámicos (definición en el texto)

- Serología positiva para Mycoplasma sp,

Chlamydia sp o Legionella sp.

Se recomienda agregar:

Eritromicina $500 \mathrm{mg}$ cada $6 \mathrm{~h} \mathrm{EV} \mathrm{o} \mathrm{VO,}$ claritromicina $500 \mathrm{mg}$ cada $12 \mathrm{~h} \mathrm{VO,} \mathrm{o}$ azitromicina $500 \mathrm{mg} /$ día VO

Ceftriaxona 2 gramos/día EV, o cefotaxima 1 gramo cada 8 horas EV

Asociado a:

Eritromicina $500 \mathrm{mg}$ cada $6 \mathrm{~h} \mathrm{EV}$, levofloxacina 0,5-1g/día EV, o moxifloxacina $400 \mathrm{mg}$ /día EV
Amoxicilina/ácido clavulánico 1.000/ $200 \mathrm{mg}$ cada $8 \mathrm{~h} \mathrm{EV}$, o amoxiclina/sulbactam 1.000/500 mg cada $8 \mathrm{~h} \mathrm{EV}$, o ampicilina/sulbactam $1.000 / 500 \mathrm{mg}$ cada $8 \mathrm{~h} \mathrm{EV}$

Asociado a:

Eritromicina $500 \mathrm{mg}$ cada $6 \mathrm{~h} \mathrm{EV}$, levofloxacina $0,5-1 \mathrm{~g}$ /día EV, o moxifloxacina $400 \mathrm{mg} /$ día $\mathrm{EV}$

* La elección de la vía de administración de los antibióticos, oral (VO) o endovenosa (EV), depende de la gravedad de la infección y funcionamiento del tracto digestivo del enfermo.

- La duración del tratamiento antimicrobiano en la NAC de gravedad moderada que requiere hospitalización, de etiología desconocida, es 7 a 10 días [C].

\section{Tratamiento antimicrobiano de la NAC grave}

En esta categoría se concentran los pacientes de alto riesgo de complicaciones y muerte ${ }^{1-4}$. La letalidad de la NAC grave manejada en la UCI fluctúa entre 17 y $50 \%{ }^{26,56}$. Los patógenos más importantes aislados en pacientes con NAC grave son $S$. pneumoniae, bacilos gramnegativos entéricos, Legionella sp, $S$. aureus, $H$. influenzae, anaerobios estrictos, C. pneumoniae, M. pneumoniae y los virus respiratorios ${ }^{26,56}$. En pacientes con daño pulmonar crónico (enfermedad pulmonar obstructiva crónica-EPOC y bronquiectasias), especialmente si tienen el antecedente de infección o colonización previa por Pseudomonas aeruginosa, debe considerarse este microorganismo en el tratamiento empírico inicial; este patógeno se asocia a mayor riesgo de muerte y se recomienda cubrir con la asociación de ceftazidima y ciprofloxacina (Tabla 3).
Se debe evitar el uso de aminoglucósidos porque no alcanzan concentraciones elevadas en el tejido pulmonar, se inactivan en medio ácido y su perfil de efectos adversos es desfavorable comparado con ciprofloxacina.

Se dispone de escasa información acerca del tratamiento de la NAC grave (grupo 4) ${ }^{57,58}$ [III]; sin embargo, algunos estudios sugieren que el tratamiento empírico inicial inapropiado se asocia a un incremento de la letalidad ${ }^{59}$ [II]. Debido a la elevada tasa de complicaciones y letalidad asociados a la NAC grave, se recomienda la administración de tratamiento antibiótico combinado vía endovenosa con prontitud una vez establecido el diagnóstico ${ }^{26}$ [D].

Un trabajo retrospectivo que evaluó varios esquemas antibióticos en adultos hospitalizados por NAC grave en UCI, demostró que la inclusión de un macrólido en el tratamiento empírico inicial parece ser segura y efectiva [III]. El tratamiento con fluoroquinolonas en un reducido grupo de pacientes también fue examinado en este estudio y no incrementó el riesgo de complicaciones y muerte ${ }^{58}$. 
Basados en estos antecedentes, se recomienda el tratamiento combinado de un agente $\beta$ lactámico o un $\beta$-lactámico con inhibidor de $\beta$ lactamasa asociado a macrólidos o fluoroquinolonas vía endovenosa (cefotaxima, ceftriaxona o amoxicilina/ácido clavulánico asociada a eritromicina, moxifloxacina o levofloxacina vía endovenosa) [C]. Se recomienda restringir el empleo de las nuevas fluoroquinolonas a pacientes con NAC grave para evitar el desarrollo de cepas resistentes y tiene ventajas sobre la eritromicina ya que se puede administrar en una o dos dosis diarias por una vena periférica y tiene menos efectos adversos asociados al uso endovenoso (flebitis) ${ }^{25}$.

\section{Recomendaciones para pacientes hospitaliza- dos con NAC grave}

- El tratamiento antibiótico empírico deber ser combinado y administrado por vía parenteral [C].

- El antibiótico prescrito debe ser administrado precozmente, idealmente antes de 4 horas de realizado el diagnóstico [C].

- En general, la duración del tratamiento antimicrobiano fluctúa entre los 10 y 14 días, dependiendo de la evolución clínica y el agente causal de la neumonía [C].

\section{Tratamiento antibiótico específico}

\section{Neumonía con agente etiológico conocido}

En la práctica clínica, sólo en 20 a $30 \%$ de los casos de NAC que requieren hospitalización se logra identificar el agente causal ${ }^{1-3,15,45}$ [II]. De acuerdo al patógeno respiratorio identificado se recomienda el tratamiento antimicrobiano específico (Tabla 4). Sin embargo, la elección del esquema antibiótico y la vía de administración deben basarse no sólo en la información microbiológica, sino además debe considerar el resultado del antibiograma, la condición general y gravedad del paciente y la posibilidad de una infección polimicrobiana.

\section{Neumonía aspirativa}

El diagnóstico clínico de neumonía aspirativa se plantea cuando existe un infiltrado radiográfico en un lóbulo o segmento pulmonar dependiente (principalmente el lóbulo inferior derecho) asociado a factores de riesgo de broncoaspiración.

Tabla 3. Tratamiento antimicrobiano empírico en situaciones especiales

- Pacientes con sospecha de neumonía aspirativa

- Régimen de elección: Ceftriaxona 1-2 g/día vía ev o cefotaxima 1 g cada 8 h vía ev.

- Régimen especial: Agregar clindamicina 600 mg cada 8 h vía ev o metronidazol 500 mg cada 8 h vía ev en las siguientes situaciones clínicas:

- Presencia de expectoración pútrida

- Enfermedad periodontal severa

- Alcoholismo

- Sospecha de aspiración gástrica mayor

- Cavitación o absceso pulmonar en la radiografía de tórax

En el cambio a la vía oral se recomienda: Clindamicina $300 \mathrm{mg}$ c/6 h, amoxicilina/ácido clavulánico 500/125 mg cada $8 \mathrm{~h}$ o $875 / 125 \mathrm{mg}$ cada $12 \mathrm{~h}$, o amoxicilina 750-1.000 $\mathrm{mg}$ cada $8 \mathrm{~h}$ asociado a metronidazol $500 \mathrm{mg}$ cada $8 \mathrm{~h}$ Duración del tratamiento: 10-14 días

- Factores de riesgo de infección por Pseudomonas aeruginosa

- Bronquiectasias

- Enfermedad pulmonar obstructiva crónica (EPOC)

- Tratamiento corticoesteroidal crónico (prednisona o equivalente $>10 \mathrm{mg} / \mathrm{día}$ )

Régimen de elección: Ceftazidima 2 g cada 8 h ev o piperacilina/tazobactam 4,5 g cada 8 h ev, asociado a ciprofloxacina 500-750 mg cada $12 \mathrm{~h}$ ev o vo.

Régimen alternativo: Imipenem $500 \mathrm{mg}$ cada $6 \mathrm{~h}$ ev o cefepime 1-2 g cada $12 \mathrm{~h}$ ev, asociado a ciprofloxacina 500-750 mg cada $12 \mathrm{~h}$ ev o vo

* La elección de la vía de administración de los antibióticos, oral (VO) o endovenosa (EV), depende de la gravedad de la infección y funcionamiento del tracto digestivo del enfermo. 
Se estima que representa entre el 5 y $15 \%$ de los casos de NAC, siendo más frecuente en adultos mayores que viven en centros geriátricos. Las condiciones clínicas de riesgo para aspirar incluyen la disfagia neurológica (ej: enfermedad de Parkinson o accidente cerebrovascular con compromiso de la musculatura orofaríngea), trastornos de la unión gastroesofágica y alteraciones anatómicas del tracto aerodigestivo superior $^{60}$. Los pacientes con neumonía aspirativa están en riesgo de desarrollar cavitación y abscesos pulmonares. Los principales patógenos involucrados son anaerobios estrictos (Bacteroides sp, Prevotella melaninogenica, Fusobacterium sp, Peptostreptococcus sp) asociado a bacterias aeróbicas como $S$. pneumoniae, $S$. aureus, $H$. influenzae y Enterobacteriaceas ${ }^{61-63}$. De acuerdo a esta información, se recomienda que el tratamiento cubra los bacilos gramnegativos entéricos y no es necesario el uso rutinario de agentes antianaeróbicos. En la mayoría de los pacientes con neumonía y riesgo de aspiración se recomienda emplear cefalosporinas de $3^{\text {a }}$ generación (cefotaxima o ceftriaxona) [C]. El uso de clindamicina aumenta los costos del tratamiento y se ha asociado a diarrea por $C$. difficile. La adición de un fármaco antianaeróbico se reserva para pacientes con neumonía aspirativa y enfermedad periodontal severa, alcoholismo, expectoración pútrida, o la presencia de una cavitación o absceso pulmonar en la radiografía de tórax $^{60}$ (Tabla 3) [C].

\section{Criterios de estabilidad clínica, cambio a tratamiento antibiótico oral y egreso hospitalario}

La mayoría de los pacientes con neumonía se estabilizan clínicamente entre el segundo y tercer día en el hospital. La estabilización clínica se produce cuando se normalizan los signos vitales, el estado mental es normal o retorna a la condición basal y mejora el intercambio gaseoso disminuyendo los requerimientos de oxígeno ${ }^{2,64,65}$ [Ia, II]. Se ha enfatizado que la estabilidad clínica debe mantenerse por lo menos durante 24 horas. Una vez que se alcanza la estabilidad clínica y el paciente puede comer o alimentarse por una sonda nasoenteral o gastrostomía se recomienda realizar el cambio de la terapia antibiótica parenteral a la vía oral (Tabla 5). Esto ocurre en la mayoría de los casos entre el tercer y quinto día de tratamiento. Se han reconocido grupos de pacientes que suelen demorar más en alcanzar la estabilidad clínica sin que esto implique una falla del tratamiento empíri- co inicial. En esta categoría se encuentran los adultos mayores, los pacientes con neumonía grave, insuficiencia cardíaca descompensada o EPOC avanzada ${ }^{2,64,65}$ [Ib] 
Tabla 4. Tratamiento antimicrobiano específico según agente causal de la NAC

Streptococcus pneumoniae: Amoxicilina $750-1.000 \mathrm{mg}$ cada $8 \mathrm{~h}$ vo, eritromicina $500 \mathrm{mg}$ cada $6 \mathrm{~h}$ vo o ev, claritromicina $500 \mathrm{mg}$ cada $12 \mathrm{~h}$ vo, cefuroxima $750 \mathrm{mg}$ cada $8 \mathrm{~h} \mathrm{ev}$, ceftriaxona $1-2 \mathrm{~g} / 24 \mathrm{~h} \mathrm{ev}$, o cefotaxima $1 \mathrm{~g}$ cada $8 \mathrm{~h}$ ev

Duración del tratamiento: 7-10 días

Mycoplasma pneumoniae y Chlamydia pneumoniae: Eritromicina 500 mg cada 6 h vo o ev, claritromicina 500 mg cada 12 h vo, tetraciclina $500 \mathrm{mg}$ cada 6 h vo, azitromicina 500 mg/día

Duración del tratamiento: 14 días (excepto con azitromicina de la que se recomiendan 5 días)

Legionella sp: Claritromicina $500 \mathrm{mg}$ cada 12 h vo, levofloxacina $500 \mathrm{mg}$ cada 12 h vo o $1 \mathrm{~g} / \mathrm{día}$ ev, moxifloxacina $400 \mathrm{mg} /$ día vo o ev, o gatifloxacina $400 \mathrm{mg} /$ día vo, asociado a rifampicina $600 \mathrm{mg}$ cada $12 \mathrm{~h}$ vo

Duración del tratamiento: 14-21 días

Haemophilus influenzae no productor de $\beta$-lactamasa: Amoxicilina $750-1.000 \mathrm{mg}$ cada $8 \mathrm{~h}$ vo

Haemophilus influenzae productor de $\boldsymbol{\beta}$-lactamasa: Amoxicilina/ácido clavulánico 500/125 mg cada 8 h o 875/ $125 \mathrm{mg}$ cada $12 \mathrm{~h}$ vo, cefuroxima $750 \mathrm{mg}$ cada $8 \mathrm{~h}$ ev, ceftriaxona 1-2 g/día ev; o cefotaxima $1 \mathrm{~g}$ cada $8 \mathrm{~h}$ ev

Duración del tratamiento: 10-14 días

Bacilos Gram negativos entéricos: Cefotaxima 1-2 g cada 8 h ev, o ceftriaxona 1-2 g/día ev Duración del tratamiento: 14-21 días

Pseudomonas aeruginosa: Ceftazidima $2 \mathrm{~g}$ cada $8 \mathrm{~h}$ ev o piperacilina/tazobactam $4,5 \mathrm{~g}$ cada $8 \mathrm{~h}$ ev, asociado a ciprofloxacina $500-750 \mathrm{mg}$ cada $12 \mathrm{~h}$ vo o ev

Duración del tratamiento: 14-21 días

Staphylococcus aureus meticilina sensible: Cloxacilina 500-1.000 mg cada 6 horas vo o ev

Staphylococcus aureus meticilina resistente: Vancomicina $1 \mathrm{~g}$ cada $12 \mathrm{~h}$ ev

Duración del tratamiento: 14-21 días

* El tratamiento puede modificarse según el antibiograma. La elección de la vía de administración de los antibióticos depende de la gravedad de la infección y funcionamiento del tracto digestivo del enfermo.

enfermedad que se han asociado a retardo en la resolución clínica de la neumonía. En realidad, esta condición clínica se confunde pero no corresponde a la categoría de fracaso de tratamiento, ya que los enfermos suelen mejorar sin necesidad de modificar el esquema antimicrobiano inicial.

- Uso de antibióticos inapropiados. Se recomienda revisar el espectro antibacteriano, la vía y dosis de los antibióticos prescritos en cada caso particular.

- Presencia de un patógeno resistente. Se recomienda revisar el antibiograma de los microorganismos aislados en los cultivos, especialmente considerando la presencia de $S$. pneumoniae resistente a $\beta$-lactámicos y macrólidos, $H$. influenzae productor de $\beta$ lactamasa, $S$. aureus meticilina resistente y bacilos gramnegativos entéricos multiresistentes.

- Patógeno no cubierto por el tratamiento empírico inicial: Se recomienda modificar el esquema antibiótico de acuerdo a los resultados de los exámenes microbiológicos, especialmente en la infección por agentes atípicos (solicitar serología para $M$. pneumoniae y $C$. pneumoniae, y antígeno urinario de L. pneumophila), S. aureus o $P$. aeruginosa (solicitar cultivos de muestras respiratorias en grupos de riesgo), Mycobacterium tuberculosis (solicitar baciloscopias y cultivo de Koch).

- Sospecha de inmunosupresión: En pacientes con factores de riesgo de inmunosupresión (ej: tratamiento corticoesteroidal, infección por VIH, quimioterapia, trasplante de órganos sólidos o precursores hematopoyéticos, etc), se recomienda la búsqueda sistemática de patógenos oportunistas tales como Pneumocystis jirovecii, Mycobacterium avium intracellulare, Nocardia asteroides y Rodococcus equi. La fibrobroncoscopia con lavado broncoalveolar y biopsia transbronquial es un procedimiento diagnóstico útil cuando se sospecha infección por patógenos oportunistas $\mathrm{o}$ inusuales. 
Tabla 5. Criterios de estabilidad clínica para decidir el cambio del tratamiento antibiótico a la vía oral y el egreso hospitalario ${ }^{2,64}$

Criterios de estabilidad clínica para decidir el cambio del antibiótico a la vía oral

- Signos vitales estables durante 24 horas: Frecuencia cardíaca $\leq 100$ latidos/min, presión arterial sistólica $\geq 90$ $\mathrm{mmHg}$, frecuencia respiratoria $\leq 24$ respiraciones/min, temperatura $<37,8^{\circ} \mathrm{C}$

- Mejoría significativa y/o resolución de los síntomas respiratorios

- Ausencia o disminución de los requerimientos de oxígeno

- Estado mental normal o retorno a la condición basal

- Tubo digestivo funcionante o capaz de ingerir el antibiótico vía oral

\section{Criterios de estabilidad clínica para decidir el alta hospitalaria}

- Haber cumplido los criterios de cambio a la vía oral

- $\mathrm{SaO}_{2} \geq 90 \%$ respirando aire ambiente o con bajo flujo de oxígeno

- Las comorbilidades deben estar compensadas

- Ausencia de factores psicosociales que contraindiquen el manejo ambulatorio

Antibióticos prescritos en el cambio a la vía oral.

Amoxicilina/ácido clavulánico 500/125 mg cada 8 h o 875/125 mg cada 12 h

Cefuroxima $500 \mathrm{mg}$ cada $12 \mathrm{~h}$

Claritromicina $500 \mathrm{mg}$ cada $12 \mathrm{~h}$

Levofloxacina $500 \mathrm{mg} /$ día

- Complicaciones de la neumonía: En el paciente que se demora en alcanzar la estabilidad clínica se recomienda la búsqueda activa de complicaciones, tales como cavitación o absceso pulmonar, empiema pleural, síndrome de distrés respiratorio agudo o infección extrapulmonar. Repetir la radiografía de tórax y/o solicitar una tomografía axial computada de tórax puede ser útil cuando se sospechan estas complicaciones en el curso de la evolución.

- Causas no infecciosas: Existen múltiples condiciones clínicas que pueden simular una neumonía tales como la insuficiencia cardíaca descompensada, tromboembolismo pulmonar, bronquiolitis obliterante con neumonía en organización, neumonía postobstructiva asociada a tumor, carcinoma bronquioloalveolar, hemorragia alveolar, linfoma, neumonía eosinofílica, sarcoidosis, daño pulmonar por fármacos, neumonitis alérgica extrínseca, neumonitis actínica y neumonitis intersticial aguda. La tomografía axial computada de tórax es útil en el diagnóstico diferencial de estas condiciones.
Cuando el paciente evoluciona mal y se cumplen los criterios de falla clínica, se recomienda la búsqueda sistemática de las posibles causas y ampliar la cobertura antibiótica, ya que en esta categoría aumenta significativamente el riesgo de muerte ${ }^{67}$. De este modo, si la terapia inicial ha sido un agente $\beta$-lactámico, se recomienda adicionar un macrólido o fluoroquinolona para cubrir los microorganismos atípicos (Mycoplasma sp, Chlamydia sp y Legionella sp), si se sospecha aspiración de contenido gástrico o existe cavitación o absceso pulmonar se recomienda adicionar un agente antianaeróbico (metronidazol o clindamicina) [D].

\section{Manejo de las complicaciones de la neumonía}

Las complicaciones específicas asociadas a la neumonía comunitaria son el efusión pleural, empiema y absceso pulmonar. El 20-60\% de los episodios de neumonía manejados en el hospital presentan efusión pleural, la mayoría evolucionan favorablemente con el tratamiento antibiótico, y se estima que cerca del $10 \%$ de los casos se complican con infección del espacio pleural 
o empiema, requiriendo drenaje $\mathrm{j}^{45,68}$ [II]. En los pacientes con efusión pleural paraneumónico se recomienda realizar siempre una toracocentesis diagnóstica e idealmente evacuadora, ya que la infección del espacio pleural aumenta la morbilidad y letalidad del enfermo ${ }^{68}$ [D]. La presencia de líquido pleural purulento, $\mathrm{pH}<7,2$, presencia de bacterias en la tinción Gram o cultivo, y lactato $>4 \mathrm{mmol} / \mathrm{l}$ permiten establecer el diagnóstico de efusión pleural a empiema, lo cual requiere un rápido y efectivo drenaje del espacio pleural. Los principales patógenos aislados en el empiema son flora mixta que incluye a S. pneumoniae, $S$. aureus, bacilos gramnegativos entéricos, y agentes anaerobios estrictos (Bacteroides melaninogenicus, Peptostreptococcus sp, Fusobacterium nucleatum). Se recomienda tratar estos casos con una cefalosporina de $3^{\text {a }}$ generación (cefotaxima o ceftriaxona) asociado a un agente antianaeróbico (metronidazol o clindamicina) [D].

La neumonía cavitada o absceso pulmonar es una complicación poco frecuente en nuestros días, que se asocia al antecedente de alcoholismo, factores de riesgo de aspiración, obstrucción bronquial por neoplasia o cuerpo extraño, y mala higiene bucal. Los principales microorganismos aislados son bacterias anaeróbicas estrictas, S. aureus y bacilos gramnegativos entéricos. La terapia antimicrobiana debe ser prolongada y se extiende hasta lograr la resolución o estabilidad radiológica. Algunos estudios sugieren que clindamicina es más efectiva que penicilina en el manejo del absceso pulmonar ${ }^{70,71}$ [Ib], y recientemente se ha demostrado la efectividad de amoxicilina/ácido clavulánico en el tratamiento de la infección pulmonar por anaerobios estrictos $^{72}$ [Ib]. Se recomienda tratar el absceso pulmonar con clindamicina $600 \mathrm{mg}$ cada 6-8 horas vía endovenosa durante 7-10 días, seguido de clindamicina $300 \mathrm{mg}$ cada 6 horas vía oral o amoxicilina/ácido clavulánico 875/125 mg cada 8-12 horas vía oral durante 4-6 semanas y/o hasta haber obtenido estabilidad de las imágenes radiológicas [B]. Examinar el papel de la fibrobroncoscopia, drenaje percutáneo y cirugía en el manejo del absceso pulmonar está fuera del alcance de esta guía clínica.

\section{Resumen}

El tratamiento antimicrobiano apropiado reduce la duración de la sintomatología asociada a la neumonía, el riesgo de complicaciones y la mortalidad. En la mayoría de los casos, no es posible identificar el agente microbiológico que ocasiona la infección y por esto el tratamiento antibacteriano se prescribe en forma empírica. En Chile, un tercio de las cepas de Streptococcus pneumoniae muestra susceptibilidad disminuida a penicilina; mientras que la resistencia a eritromicina fluctúa entre 10$15 \%$ y a cefotaxima entre $2-10 \%$. Se recomienda clasificar a los pacientes con neumonía adquirida en la comunidad en cuatro categorías de riesgo: Grupo 1: pacientes bajo 65 años de edad, sin comorbilidad de manejo ambulatorio. Tratamiento: amoxicilina 1 gramo cada 8 horas vía oral durante 7 días. Grupo 2: pacientes sobre 65 años de edad y/o con comorbilidad de manejo ambulatorio. Tratamiento: amoxicilina/ácido clavulánico 500/125 mg cada 8 horas ó $875 / 125 \mathrm{mg}$ cada 12 horas, o cefuroxima $500 \mathrm{mg}$ cada 12 horas vía oral durante 7 días. Grupo 3: pacientes hospitalizados en sala de cuidados generales que tienen criterios de gravedad moderada. Tratamiento: ceftriaxona 1-2 g/día o cefotaxima $1 \mathrm{~g}$ cada 8 horas EV durante 7-10 días. Grupo 4: pacientes con neumonía grave adquirida en la comunidad que deben ser manejados en la UCI. Tratamiento: ceftriaxona $2 \mathrm{~g} /$ día o cefotaxima 1 g cada 8 horas EV asociado a eritromicina $500 \mathrm{mg}$ cada $6 \mathrm{~h}$, levofloxacina 500-1.000 mg/día, o moxifloxacina 400 mg/día EV durante 10-14 días. En presencia de alergia o fracaso de tratamiento con agentes $\beta$-lactámicos y/o serología positiva para Mycoplasma, Chlamydia o Legionella sp se recomienda agregar: eritromicina $500 \mathrm{mg}$ cada $6 \mathrm{~h} \mathrm{EV} \mathrm{o}$ $\mathrm{VO}$, claritromicina $500 \mathrm{mg}$ cada $12 \mathrm{~h} \mathrm{VO}$, o azitromicina $500 \mathrm{mg} /$ día VO.

\section{Bibliografía}

1.- British Thoracic Society Standards of Care Committee. British Thoracic Society guidelines for the management of community-acquired pneumonia in adults. Thorax 2001; 56 (Suppl IV): 1-64.

2.- Niederman M S, Mandell L A, Anzueto A, Bass J B, Broughton W A, Campbell G D, et al. Guidelines for the management of adults with community-acquired pneumonia. Diagnosis, assessment of severity, antimicrobial therapy, and prevention. Am J Respir Crit Care Med 2001; 163: 1730-54.

3.- Mandell L A, Bartlett J G, Dowell S F, File T M Jr, Musher D M, Whitney C. Update of practice guidelines for the management of community-acquired pneumonia in immunocompetent adults. Clin Infect Dis 2003; 37: 1405-33.

4.- Update to the Latin American Thoracic Association (ALAT) recommendations on community acquired pneumonia. Arch Bronconeumol 2004; 40: 364-74.

5.- Marrie T J, Lau C Y, Wheeler S L, Wong C J, Vandervoort M K, Feagan B G. A controlled trial of a critical pathway for treatment of community-acquired pneumonia. CAPITAL Study Investigators. Community-Acquired Pneumonia Intervention Trial Assessing Levofloxacin. JAMA 2000; 283: 749-55.

6.- Menendez R, Ferrando D, Valles J M, Vallterra J. Influence of deviation from guidelines on the outcome of community-acquired pneumonia. Chest 2002; 122 : 612-7.

7.- Malone D C, Shaban H M. Adherence to ATS guidelines for hospitalized patients with community-acquired 
acquired lower respiratory tract infection in a general hospital: open, randomised controlled trial. BMJ 1995; 310: $1360-2$.

37.- Anderson G, Esmonde T S, Coles S, Macklin J, Carnegie C. A comparative safety and efficacy study of clarithromycin and erythromycin stearate in community-acquired pneumonia. J Antimicrob Chemother 1991; 27 (Suppl A): 117-24.

38.- Chien S M, Pichotta P, Siepman N, Chan C K. Treatment of community-acquired pneumonia. A multicenter, double-blind, randomized study comparing clarithromycin with erythromycin. Canada-Sweden Clarithromycin-Pneumonia Study Group. Chest 1993; 103: 697-701.

39.- O'doherty B, Muller O. Randomized, multicentre study of the efficacy and tolerance of azithromycin versus clarithromycin in the treatment of adults with mild to moderate community-acquired pneumonia. Azithromycin Study Group. Eur J Clin Microbiol Infect Dis 1998; 17: 828-33.

40.- Rovira E, Martínez-Moragon E, Belda A, Gonzalvo F, Ripolles F, Pascual J M. Treatment of communityacquired pneumonia in outpatients: randomized study of clarithromycin alone versus clarithromycin and cefuroxime. Respiration 1999; 66: 413-8.

41.- Vergis E N, Indorf A, File T M Jr, Phillips J, Bates J, Tan J, et al. Azithromycin vs cefuroxime plus erythromycin for empirical treatment of communityacquired pneumonia in hospitalized patients: a prospective, randomized, multicenter trial. Arch Intern Med 2000; 160: 1294-300.

42.- File T M Jr, Segreti J, Dunbar L, Player R, Kohler R, Williams R R, et al. A multicenter, randomized study comparing the efficacy and safety of intravenous and/ or oral levofloxacin versus ceftriaxone and/or cefuroxime axetil in treatment of adults with community-acquired pneumonia. Antimicrob Agents Chemother 1997; 41: 1965-72.

43.- Van Den Brande P, Vondra V, Vongel F, Schlaeffer F, Staley H, Holmes C. Sequential therapy with cefuroxime followed by cefuroxime axetil in community-acquired pneumonia. Chest 1997; 112: 406-15.

44.- Bjerre L M, Verheij T J, Kochen M M. Antibiotics for community acquired pneumonia in adult outpatients. Cochrane Database Syst Rev 2004; (2): CD002109.

45.- Saldías F, Mardonez J M, Marchesse M, Viviani P, Farías G, Díaz A. Neumonía adquirida en la comunidad en el adulto hospitalizado. Cuadro clínico y factores pronósticos. Rev Méd Chile 2002; 130: 1373-82.

46.- Rioseco M L, Riquelme R. Neumonía neumocócica bacterémica en 45 adultos inmunocompetentes hospitalizados. Cuadro clínico y factores pronósticos. Rev Méd Chile 2004; 132: 588-94.

47.- Fernández P, San Martín L. Neumonía adquirida en la comunidad: Terapia secuencial de cefalosporina intravenosa a cefalosporina oral. Rev Méd Chile 2000; 128: $267-72$

48.- Oosterheert J J, Bonten M J, Hak E, Schneider M M, Hoepelman I M. How good is the evidence for the recommended empirical antimicrobial treatment of patients hospitalized because of community-acquired pneumonia? A systematic review. J Antimicrob Chemother 2003; 52: 555-63.

49.- Frei C R, Koeller J M, Burgess D S, Talbert R L, Johnsrud M T. Impact of atypical coverage for patients with community-acquired pneumonia managed on the medical ward: results from the United States
Community-Acquired Pneumonia Project. Pharmacotherapy 2003; 23: 1167-74.

50.- Rabagliati R, Benítez R, Fernández A, Gaete P, Guzmán A M, García P, et al. Reconocimiento de influenza-A como etiología de síndrome febril e insuficiencia respiratoria en adultos hospitalizados durante brote en la comunidad. Rev Méd Chile 2004; 132: 317-24.

51.- Douglas R G Jr. Prophylaxis and treatment of influenza. N Engl J Med 1990; 322: 443-50.

52.- Gubareva L V, Kaiser L, Hayden F G. Influenza virus neuraminidase inhibitors. Lancet 2000; 355: 827-35.

53.- Houck P M, Bratzler D W, Nsa W, Ma A, Bartlett J G. Timing of antibiotic administration and outcomes for Medicare patients hospitalized with communityacquired pneumonia. Arch Intern Med 2004; 164: 637 44.

54.- Meehan T P, Fine M J, Krumholz H M, Scinto J D, Galusha D H, Mockalis J T, et al. Quality of care, process, and outcomes in elderly patients with pneumonia. JAMA 1997; 278: 2080-4.

55.- Battleman D S, Callahan M, Thaler H T. Rapid antibiotic delivery and appropriate antibiotic selection reduce length of hospital stay of patients with community-acquired pneumonia: link between quality of care and resource utilization. Arch Intern Med 2002; 162: $682-8$.

56.- Díaz A, Álvarez M, Callejas C, Rosso R, Schnettler K, Saldías F. Cuadro clínico y factores pronósticos de la neumonía comunitaria grave en adultos hospitalizados en la Unidad de Cuidados Intensivos. Arch Bronconeumol 2005; 41: 20-6.

57.- Romanelli G, Cravarezza P, Pozzi A, Franchino L, Ravizzola G, Zulli R, et al. Carbapenems in the treatment of severe community-acquired pneumonia in hospitalized elderly patients: a comparative study against standard therapy. J Chemother 2002; 14: 60917.

58.- Rello J, Catalán M, Díaz E, Bodi M, Álvarez B. Associations between empirical antimicrobial therapy at the hospital and mortality in patients with severe community-acquired pneumonia. Intensive Care Med 2002; 28: 1030-5.

59.- Torres A, Serra-Batlles J, Ferrer A, Jímenez P, Celis R, Cobo E, et al. Severe community-acquired pneumonia. Epidemiology and prognostic factors. Am Rev Respir Dis 1991; 144: 312-8.

60.- Marik P E. Aspiration pneumonitis and aspiration pneumonia. N Engl J Med 2001; 344: 665-71.

61.- Cassiere H A, M N. Aspiration pneumonia, lipoid pneumonia, and lung abcess. In Baum GL, Crapo JD, Celli BR, Karlinsky JD eds. Texbook of pulmonary diseases. 6th ed. Vol 1. Philadelphia: Lippincott-Raven. 1998: 645-55.

62.- Mier L, Dreyfuss D, Darchy B, Lanore J J, Djedaini $\mathrm{K}$, Weber $\mathrm{P}$, et al. Is penicillin $\mathrm{G}$ an adequate initial treatment for aspiration pneumonia? A prospective evaluation using a protected specimen brush and quantitative cultures. Intensive Care Med 1993; 19: 279-84.

63.- Marik P E, Careau P. The role of anaerobes in patients with ventilator-associated pneumonia and aspiration pneumonia: a prospective study. Chest 1999; 115: 17883.

64.- Rhew D C, Tu G S, Ofman J, Henning J M, Richards M S, Weingarten S R. Early switch and early discharge strategies in patients with community-acquired pneumonia: a meta-analysis. Arch Intern Med 2001; 161: $722-7$ 
65.- Ramírez J A, Bordon J. Early switch from intravenous to oral antibiotics in hospitalized patients with bacteremic community-acquired Streptococcus pneumoniae pneumonia. Arch Intern Med 2001; 161: 848-50.

66.- Halm E A, Fine M J, Kapoor W N, Singer D E, Marrie T J, Siu A L. Instability on hospital discharge and the risk of adverse outcomes in patients with pneumonia. Arch Intern Med 2002; 162: 1278-84.

67.- Arancibia F, Ewig S, Martínez J A, Ruiz M, Bauer T, Marcos M A, et al. Antimicrobial treatment failures in patients with community-acquired pneumonia: causes and prognostic implications. Am J Respir Crit Care Med 2000; 162: 154-60.

68.- Varkey B, Rose H D, Kutty C P, Politis J. Empyema thoracis during a ten-year period. Analysis of 72 cases and comparison to a previous study (1952 to 1967).
Arch Intern Med 1981; 141: 1771-6.

69.- Wehr C J, Adkins R B Jr. Empyema thoracis: a tenyear experience. South Med J 1986; 79: 171-6.

70.- Levison M E, Mangura C T, Lorber B, Abrutyn E, Pesanti E L, Levy R S, et al. Clindamycin compared with penicillin for the treatment of anaerobic lung abscess. Ann Intern Med 1983; 98: 466-71.

71.- Gudiol F, Manresa F, Pallares R, Dorca J, Rufi G, Boada $\mathrm{J}$, et al. Clindamycin vs penicillin for anaerobic lung infections. High rate of penicillin failures associated with penicillin-resistant Bacteroides melaninogenicus. Arch Intern Med 1990; 150: 2525-9.

72.- Fernández-Sabe N, Carratala J, Dorca J, Roson B, Tubau F, Manresa F, et al. Efficacy and safety of sequential amoxicillin-clavulanate in the treatment of anaerobic lung infections. Eur J Clin Microbiol Infect Dis 2003; 22: $185-7$

Correspondencia a:

Alejandro Díaz F

alediazf@hotmail.com 